\title{
Características de la micro, pequeña y mediana empresa asociadas a los tipos de tecnologías de comunicación y uso de medios sociales
}

\section{Characteristics of the Micro, Small, and Medium Business Tied to the Types of Communication Technologies and Use of Social Media}

Luis Giovanny Ortegón Cortázar (Colombia)

Universidad de Valencia giova900@gmail.com
Leonardo Ortegón Cortázar (Colombia)

Institución Universitaria Politécnico Grancolombiano

lortegon@poligran.edu.co

\section{Resumen}

La dirección en la empresa y su gestión en internet posee un especial interés e importancia, principalmente manifestado en las revistas y periódicos actuales,

\section{Abstract}

Company guidance and its management on the Internet are very interesting and important topics covered in current magazines and newspapers, as well as
FECHA DE RECEPCIÓN:

FECHA DE REVISIÓN:

APROBACIÓN:
22 de abril de 2014

28 de mayo de 2014

16 de junio de 2014
PARA CItAR ESTE artículo / to CITE thIS ARTICle

Ortegón Cortázar, L. G. y Ortegón Cortázar, L.

(2014). Características de la micro, pequeña y mediana empresa asociadas a los tipos de tecnologías de comunicación y uso de medios sociales. Poliantea, 10(18), pp. 85-110. 
y en menor medida en la literatura académica. Esta proliferación de nuevas tecnologías ha facilitado la revolución en las comunicaciones en diferentes medios, incluso el uso y la presencia de la empresa en medios sociales en la web. En este sentido, se presenta un resultado de investigación concerniente a explorar el uso de medios de comunicación en línea y de redes sociales y sus relaciones con 1) el tipo de empresa y 2) la apropiación de tecnologías digitales por parte de un conjunto de micro, pequeñas y medianas empresas en Bogotá. Para tal propósito se realizaron 191 encuestas a empresas micro, pequeñas y medianas. Los resultados indican una adaptación de las empresas a las necesidades tecnológicas modernas en el campo de la comunicación. Como conclusión, se evidenció que las empresas pueden mejorar la efectividad de sus programas de marketing con un mayor entendimiento de los recursos y herramientas tecnológicas libres o licenciadas, disponibles y adaptadas al interés de cada empresa.

Palabras clave: pyme, apropiación de TIC, presencia en redes sociales, comunicación digital. in academic literature. This proliferation of new technologies has enabled a communication revolution in different media including the use and presence of the company in social media on the web. This article presents a research regarding the use of communication media and social network online as well as their relationships with: 1) the type of business and 2) the appropriation a group of micro, small, and medium businesses in Bogotá makes of digital technologies. In order to do this, we carried out 191 surveys applied to micro, small, and medium businesses, and the results indicate that companies adapt to modern technological needs within the communication field. As a conclusion, it was possible to evidence that businesses can improve the effectiveness of their marketing programs by better understanding their resources and the free or licensed technological tools, which are available and adapted to the interest of each company.

Keywords: SMBs, TIC appropriation, social network presence, digital communication. 


\section{Características de la micro, pequeña y mediana empresa asociadas a los tipos de tecnologías de comunicación y uso de medios sociales}

\section{Characteristics of the Micro, Small, and Medium Business Tied to the Types of Communication Technologies and Use of Social Media}

\section{Luis Giovanny Ortegón Cortázar (Colombia) Universidad de Valencia Doctorando en Tecnologías de la Informa- ción, Comunicaciones y Computación en la Universidad de Valencia (España). giova900@gmail.com}

\author{
Leonardo Ortegón Cortázar (Colombia) \\ Institución Universitaria Politécnico Grancolombiano \\ Doctorando en Marketing, magíster en Psicología \\ del Consumidor. Profesor de la Maestría en Gerencia \\ Estratégica de Mercadeo de la Institución Universitaria \\ Politécnico Grancolombiano, Bogotá (Colombia). \\ lortegon@poligran.edu.co
}

\section{Introducción}

La revolución tecnológica ocurrida en el último cuarto del siglo XX ha tenido impactos económicos y sociales que han afectado de manera importante la actividad empresarial y su relación con los consumidores (Piñero y De Maya, 2002). Las empresas han incorporado los recientes avances tecnológicos para diseñar acciones de comunicación y marketing más efectivas, utilizando como referencia la característica más revolucionaria de estos avances: la interactividad. En este sentido, la comunicación global se ha desplazado 
a múltiples plataformas y dispositivos digitales (Santomier, 2008), siendo internet la forma más difundida para lograr esta comunicación entre la empresas y sus grupos de interés, optimizando los procesos de contacto con clientes, proveedores y otros colaboradores en general.

Dados los anteriores argumentos, el propósito de este artículo consiste en explorar el uso de medios de tecnologías de comunicación en línea $\mathrm{y}$ medios sociales por parte de un conjunto de micro, pequeñas y medianas empresas en Bogotá, para determinar relaciones con 1) las características de la empresa y 2) la apropiación de las principales tecnologías digitales por parte de los empleados.

Como antecedentes a esta situación, se resalta la importancia y uso de internet como un medio de comunicación cada vez más empleado por las empresas para difundir sus mensajes publicitarios, en buena medida por los reducidos costos asociados a la ejecución e inserción de los anuncios (San José Cabezudo, Gutiérrez Arranz y Gutiérrez Cillán, 2004), como por la facilidad de gestionar comunicación entre sus propios colaboradores. Complementariamente, Palacio, Santana y Delia (2010) señalan la relevancia e incremento del nivel de penetración de internet como medio de comunicación en la sociedad en los últimos años, alcanzando un nivel de usuarios de internet hoy en día superior a 50 \% (Colombia Navega, Reporte 2011-2012). Por su parte, Ghose y Dou (1998) y Simó, Alcañiz, y Mafé (2004) afirman que internet representa un gran potencial para las comunicaciones de marketing hacia el futuro, ya que va a suponer una gran revolución en los hábitos de compra, lo que a su vez hará que se modifique por completo la relación existente entre el consumidor y los diferentes establecimientos comerciales tradicionales, y para el presente caso, un especial interés para conocer las relaciones con la empresa moderna y su nivel de apropiación de la tecnología digital.

\section{Marco teórico}

Según Klein (2003), la presencia que tiene una empresa en internet se justifica también por el potencial de proveer información completa y actualizada de los consumidores, así como por la creación de experiencias virtuales con los productos. La tendencia de comunicación en línea ha sido estudiada en diversos informes (Cyr, Kindra y Dash, 2008), donde se percibe un incremento sobre el 
número de usuarios interconectados. Colombia no es ajena a esta tendencia (Mora y Hurtado, 2011), y así es como en la actualidad se calcula que hay más de cinco millones de suscriptores de internet (fijo o móvil), traduciéndose en más de treinta y cinco millones de personas navegando por la red. Se subraya, además, que un crecimiento de $10 \%$ en la penetración de internet puede incrementar el crecimiento de un país en $1 \%$ (Valero, 2011). En complemento, Ryan y Jones (2009) señalan que no solo hay más personas conectadas, sino que, además, usan internet más seguido, por periodos más largos y realizan más actividades en la red. Dando como resultado que la penetración del mercadeo por canales digitales se haya incrementado a gran velocidad en los últimos años, incluso, la utilización de herramientas virtuales de comunicación, como voz IP (voz sobre protocolo de internet, voice over $I P)$, correo electrónico y mensajería interna, motivo por el cual deben investigarse estas características en la empresa moderna.

Esta proliferación de nuevas tecnologías ha facilitado la revolución en las comunicaciones en diferentes medios, incluso, el uso y presencia de la empresa en medios sociales en la web, con lo cual estas se enfrentan al reto de obtener no solo la atención, sino también la aprobación de sus usuarios para establecer una experiencia de comunicación comercial, lo que, de acuerdo con Vargo y Lusch (2004), ha generado una transición en la lógica del mercadeo, de una visión centrada en los bienes tangibles, hacia una nueva visión centrada en el valor intangible y las experiencias que la empresa o marca pueda construir en la mente de los consumidores, manifestadas en experiencias y comportamientos sensoriales de preferencia o evitación. Con esta nueva visión, Vargo y Lusch (2004) afirman que el valor debe ahora ser definido y cocreado con el usuario en lugar de ser "entregado" o "enviado" a este por parte de la empresa, por lo cual se deben buscar estrategias o métodos para recibir una retroalimentación de la comunicación comercial en línea. Tal idea es precisamente considerada en la perspectiva de la web 2.0, donde surge un espacio bidireccional que permite a sus públicos opinar y comunicarse con ellas: blogs, YouTube, redes sociales, son solo un ejemplo de este nuevo territorio que conquistar por las empresas (García, 2011). 


\section{Sector empresarial colombiano}

Existe consenso acerca de la importancia del sector de las pequeñas y medianas empresas para el desarrollo económico y el equilibrio social en Colombia, por su contribución a la generación de riqueza, empleo, crecimiento económico, bienestar y mejora en la distribución del ingreso (Rodríguez, 2012). Dichas empresas agrupan gran variedad de unidades económicas de diferente naturaleza y nivel de desarrollo. La información a partir de la cual se puede hacer una aproximación a estas proviene de diversas fuentes con diferentes metodologías y contenidos temáticos, principalmente informes del Ministerio de Tecnologías de la Información y las Comunicaciones, del Ministerio de Industria, Comercio y Turismo y de estudios de entidades, como el Departamento Nacional de Planeación (DNP), la Asociación Nacional de Instituciones Financieras (ANIF), el Departamento Administrativo
Nacional de Estadística (DANE) y otras entidades estatales y educativas. Toda esta información dificulta un análisis estandarizado o uniforme de la situación de las micro, pequeñas y medianas empresas en Colombia. A continuación se exponen características generales de este sector.

La categorización de las empresas en Colombia surge con la Ley MiPyme (Ley 590 de 2000), aprobada por el Congreso de Colombia y modificada por la Ley 905 de 2004. En ella se establece como "micro, pequeña y mediana empresa, toda unidad de explotación económica, realizada por persona natural o jurídica, en actividades empresariales, agropecuarias, industriales, comerciales o de servicios, rural o urbana, ..." que cumpla con los criterios de activos y número de empleados. En la tabla 1 se presentan las características de dicha categorización, aclarando que, en caso de existir discrepancias, prevalece el factor activos (tabla 1).

Tabla 1. Categorización de tamaño de empresa en Colombia

\begin{tabular}{lcccc}
\hline \multirow{2}{*}{ Tamaño } & \multicolumn{2}{c}{ Empleados } & \multicolumn{2}{c}{ Activos (en salarios mínimos)* } \\
\cline { 2 - 5 } & Entre & $\mathbf{y}$ & Entre & y \\
\hline Micro & 1 & 10 & 1 & 500 \\
Pequeña & 11 & 50 & 501 & 5000 \\
Mediana & 51 & 200 & 5001 & 30000 \\
Grande & 201 & 0 más & 30001 & 0 más \\
\hline
\end{tabular}

"smmlv: salario mínimo mensual legal vigente, que para 2012 corresponde a $\$ 589500$ (aprox. $€ 244.86$ ), siendo $€$ equivalente a $\$ 2407.45$ (03/06/2013).

Fuente: Elaboración propia. 
Al intentar relacionar dicha caracterización de tamaño de empresa con el uso de medios sociales de las empresas, no se encuentra información específica que resuelva dicho interrogante. Sin embargo, Canals (2001) señala al menos que el tamaño de la red y no de la empresa es un factor importante para el éxito a mediano plazo, sin indicar cómo su tamaño (microempresa, pequeña y mediana) puede estar relacionado con dicho propósito en su obra $\mathrm{La}$ estrategia de la empresa en la era de internet. En complemento, el Censo General 1990-2005 realizado por el DANE describe que las micro, pequeñas y medianas empresas representan casi $90 \%$ de las empresas del país. Solo las microempresas representan aproximadamente $96 \%$ del total, de este valor $56.1 \%$ se dedica a la actividades de comercio, $32.8 \%$ a la prestación de servicios, y solo $11 \%$ a la industria. La importancia de las actividades de comercio se debe a la menor inversión de capital que hay que invertir para iniciar el negocio, ya que generalmente el retorno del capital es más rápido, en comparación con la industria, que requiere una gran inversión inicial para la puesta en funcionamiento de la actividad. Al indagar el estado del índice de apropiación de tecnologías de la información y de la comunicación (uso de computadores, uso de internet y presencia en la web), se logró construir una tabla comparativa (tabla 2), advirtiendo que, según la consulta realizada, las fuentes más recientes conseguidas para evidenciar dichos indicadores fue un informe del DANE de 2008. A la fecha, no se encontró información disponible en línea específica a estas características de tamaño y sector en las micro, pequeñas y medianas empresas. La información disponible reciente obedece a estudios de usuarios, categorías de mercado o de la industria colombiana respecto de la telefonía móvil e internet.

Tabla 2. Nivel de apropiación de tecnologías de comunicación por tamaño de la empresa

\begin{tabular}{ccc}
\hline & Tipo de uso de tecnología & Porcentaje de uso (\%) \\
\hline Microempresas & \\
& Uso de computadores & 13.20 \\
Uso de internet & 7.20 \\
Presencia en la web & 1.90
\end{tabular}

Pequeñas 
(Continuación)

\begin{tabular}{ccc}
\hline & Tipo de uso de tecnología & Porcentaje de uso (\%) \\
\hline Uso de computadores & 96.15 \\
Uso de internet & 79.35 \\
Presencia en la web & 22.95 \\
& & \\
Uedianas & Uso de computadores & 99.80 \\
& Uso de internet & 99.10 \\
& Presencia en la web & 59.55 \\
\hline
\end{tabular}

Fuente: http://www.dane.gov.co/files/investigaciones/boletines/tic/pres_tic_agos08.pdf

Se observa en la tabla 2 que exis- En este caso, la diferencia más signiten brechas grandes entre el acceso y ficativa se presenta con las microemuso de las tecnologías de comunica- presas, sin superar $15 \%$ su nivel de ción (uso de computadores, uso de apropiación. Complementariamente, internet y presencia en la web) y entre también se indagó el nivel de apropialos tipos de empresa definidos por el ción de tecnologías de comunicación tamaño (micro, pequeña y mediana). en la empresa por tipo de actividad.

Tabla 3. Nivel de apropiación de tecnologías de comunicación por tipo de actividad de la empresa (2008)

\begin{tabular}{lcc}
\hline & Tipo de uso de tecnología & Porcentaje de uso (\%) \\
\hline Servicios & Uso de computadores & 98.3 \\
& Uso de internet & 96.7 \\
& Presencia en la web & 54.6 \\
Comercio & & \\
& Uso de computadores & 97.0 \\
& Uso de internet & 93.40 \\
Industria & Presencia en la web & 31.8 \\
& & \\
& Uso de computadores & 93.7 \\
& Uso de internet & 88.7 \\
\hline
\end{tabular}

Fuente: http://www.dane.gov.co/files/investigaciones/boletines/tic/pres_tic_agos08.pdf 
Se observa en la tabla 3 que todos los sectores tienen un nivel de apropiación de computadores e internet superior a $80 \%$, exceptuando la presencia en la web, en cuyo caso el sector de servicios lidera la participación con $54.6 \%$. Por lo tanto, dicha característica de presencia en la web debe ser revisada con mayor detalle en el contexto de la comunicación.

Sicilia, Ruiz y Munuera (2005) señalan que la página web se sitúa como la herramienta de publicidad interactiva más importante en la actualidad. Asimismo, Ryan y Jones (2009) establecen que la página web es una pieza vital para la empresa, hacia la cual todas las otras actividades de comunicación en línea dirigen sus esfuerzos. Nuevamente, al querer determinar estudios previos relacionados con la disposición de páginas web y el uso de medios sociales y de tecnologías de comunicación en línea en Colombia, no existe información específica al respecto.

Otra variable de exploración proveniente de la caracterización de las empresas en Colombia es la antigüedad; al respecto y según la búsqueda efectuada; tampoco se encuentra disponible información formal y específica que relacione la antigüedad de la empresa con el uso de los medios sociales y de tecnologías de comunicación en línea en Colombia. Sobre esto es de aclarar que sí existe información de la historia y evolución de las redes sociales en Colombia, mas no de acuerdo con la antigüedad de la empresa en su implementación. Todos los anteriores argumentos permiten formular las siguientes hipótesis.

- H1: se espera que exista una relación entre las características de la empresa (tamaño, tipo de actividad, antigüedad y presencia en internet mediante un sitio web) y el uso de la empresa de medios sociales.

En complemento a dicha hipótesis y para profundizar en el uso de tecnologías de comunicación para la empresa, Sandulli et al. (2006) destacan la existencia de una relación positiva entre la inversión en tecnologías de la información y la productividad. En este caso, la inversión en tecnologías de la información desde la comunicación de la empresa puede evidenciarse en los niveles de apropiación de las principales tecnologías digitales. $\mathrm{Al}$ respecto, el Ministerio de Comunicaciones, a través de su informe de Política para la promoción 
en el acceso y uso de TIC en micro, pequeñas y medianas empresas colombianas (2009), realizó una clasificación de acuerdo con el tipo de comunicaciones presentes en la micro, pequeña y mediana empresa. Dicha clasificación propone tres tipos de comunicaciones que se articulan al presente trabajo:

- Comunicaciones básicas: uso de fax, teléfono fijo y teléfono móvil.

- Tecnología informática básica: computador/es conectado/s a una impresora, con software básico sin internet.

- Comunicaciones avanzadas (en línea): navegación en internet, uso de correo electrónico, intranet, voz sobre internet.

- H2a: se espera que exista una relación entre las características de la empresa (tamaño, tipo de actividad, antigüedad y presencia en internet mediante un sitio web) y el tipo de tecnologías de comunicaciones utilizadas en su gestión corporativa (comunicaciones básicas, comunicaciones de informática básica y comunicaciones avanzadas en línea). Esta H2 permite proyectar otros cuestionamientos importantes $\mathrm{e}$ igualmente fundamentados en Política para la promoción en el acceso y uso de TIC en micro, pequeñas y medianas empresas colombianas (2009). En dicho documento se describe que el tamaño de la empresa tiene una relación con la apropiación de las principales tecnologías digitales, evidenciado con la posibilidad de uso de computadores e internet en sus procesos productivos; es decir, posibilidad de que los empleados utilicen computadores "sin internet" o computadores "con acceso a internet". Al respecto, el informe reporta que $13.2 \%$ de las microempresas usa computadores y $7.2 \%$ internet.

- H2b: se espera que exista una relación entre la apropiación de las principales tecnologías digitales (posibilidad de uso de computadores e internet a empleados) y el tipo de tecnologías de comunicaciones utilizadas por las empresas en su gestión corporativa (comunicaciones básicas, comunicaciones de informática básica y comunicaciones avanzadas en línea).

Finalmente, y de acuerdo con los objetivos del trabajo y la revisión bibliográfica efectuada, 
se propuso una cuarta hipótesis para investigar la relación entre el nivel de acceso de las principales tecnologías digitales por parte de las empresas y el uso de comunicaciones mediante medios sociales.

- H3: se espera que exista una relación entre la apropiación de las principales tecnologías digitales (posibilidad de uso de computadores e internet a empleados) y el uso de la empresa de medios sociales de comunicación (Facebook, Twitter, blogs y YouTube).

Estas cuatro hipótesis, propuestas a partir de la revisión de la literatura y el estado de estudios aplicados que resuelvan los interrogantes planteados, señalan el valor añadido de esta investigación, pues, como se indicó, no existe información (por lo menos disponible en línea) acerca de dichas asociaciones empíricas de las variables. Desde este punto de vista, el análisis de los factores de empresa asociados a las tecnologías de comunicación y presencia en la web, incluso, los medios sociales, también pueden brindar un acercamiento para entender la realidad del mercado empresarial colombiano desde el punto de vista de la gestión de sus comunicaciones. Finalmente, para comprobar dichas presunciones, se diseñó un estudio de carácter descriptivo, el cual se detalla a continuación.

\section{Método}

El propósito de este estudio fue explorar el uso de medios de comunicación en línea y de redes sociales por parte de un conjunto de micro, pequeñas y medianas empresas en Bogotá para determinar relaciones con 1) las características de la empresa y 2) el nivel de apropiación de las principales tecnologías digitales (manifestado en posibilidad de uso de computadores e internet a empleados). Dicho propósito se encuentra enmarcado en la línea de investigación en publicidad Planificación de medios en línea desde el comportamiento de la empresa en Bogotá. Para tal propósito se escogió la industria de mipymes en Colombia con cerca de mil registros conseguidos con la plataforma electrónica BPR Benchmark, plataforma virtual privada que ofrece servicios de información de negocios y perfiles de compañías. Posteriormente, se realizó un contacto telefónico con las empresas para indagar la tipología de medios tecnológicos de comunicación que utilizan, el nivel de apropiación de las 
principales tecnologías digitales (uso de computadores e internet), el uso de medios sociales y otras características de la propia empresa, como tamaño, antigüedad y sector en el que se encuentran. A continuación se describe con detalle la metodología utilizada.

\section{Población}

El conjunto de datos resultante corresponde a 191 encuestas telefónicas efectivas, las cuales tienen proporción con una tasa de respuesta de $19.1 \%$ sobre el total de llamadas realizadas. El bajo tamaño muestral conseguido obedece a que los empresarios son reacios y desconfiados al compartir información que, muchas veces, consideran confidencial para sus compañías, y ya que consideran que este tipo de estudios no les beneficia a sus organizaciones, es común que no destinen tiempo a este tipo de actividades. Igualmente, dentro de las características de la muestra, se encuentra que $57.6 \%$ de las empresas encuestadas pertenece al sector de los servicios, mientras que $23.6 \%$ realiza actividades de comercio, y $17.8 \%$ de transformación de materias primas. Las actividades de las empresas encuestadas son prestación de servicios de asesorías en arquitectura, construcción, consultorías jurídicas, transporte privado, educación y actividades de ocio, entre otras.

\section{Instrumento}

El instrumento empleado en esta investigación corresponde a un cuestionario estructurado aplicado mediante encuesta telefónica asistida. Durante el diseño se contó con la participación de un académico y un empresario, quienes ayudaron a construir y validar el instrumento. Las variables indagadas se observan en la tabla 4.

Tabla 4. Lista de variables

\begin{tabular}{l} 
Variables \\
\hline Actividad de la empresa \\
Antigüedad de la empresa \\
Tamaño de la empresa \\
La empresa tiene sitio web \\
Nombres de redes sociales virtuales que utiliza la empresa \\
Tipo de tecnologías (medios) de comunicación que utiliza la \\
empresa en su gestión (comunicaciones básicas/comunicaciones \\
avanzadas en línea) \\
Apropiación de las principales tecnologías digitales (porcentaje de \\
empleados que utilizan computadores/porcentaje de empleados \\
que utilizan internet en el trabajo)
\end{tabular}

Fuente: Elaboración propia. 


\section{Resultados}

Para el análisis de resultados, y con- donde, posteriormente al proceso de forme al objetivo del presente trabajo, depuración de la información, se prose empleó un análisis descriptivo en- cedió a realizar un análisis descriptifocado a medir las asociaciones entre vo y luego de relaciones bivariantes las variables investigadas. El paquete con el estadístico chi-cuadrado. Los estadístico utilizado para analizar la resultados descriptivos se muestran información fue SPSS 19.0 (Statisti- en la tabla 5.

cal Package for the Social Sciences),

Tabla 5. Resultados descriptivos de variables

\begin{tabular}{|c|c|c|}
\hline Variable & Recuento & Porcentaje (\%) \\
\hline \multicolumn{3}{|l|}{ Actividad de la empresa } \\
\hline Industria & 34 & 17.8 \\
\hline Servicios & 110 & 57.6 \\
\hline Comercio & 45 & 23.6 \\
\hline Otro (agricultura) & 1 & 0.5 \\
\hline N. R. & 1 & 0.5 \\
\hline Total & 191 & \\
\hline \multicolumn{3}{|l|}{ Antigüedad de la empresa } \\
\hline Menos de 5 años & 41 & 21.5 \\
\hline Entre 5 y 10 años & 55 & 28.8 \\
\hline Entre 10 y 25 años & 56 & 29.3 \\
\hline Más de 25 años & 39 & 20.4 \\
\hline Total & 191 & \\
\hline \multicolumn{3}{|l|}{ Tamaño de la empresa } \\
\hline Microempresa & 36 & 18.8 \\
\hline Pequeña & 65 & 34.0 \\
\hline Mediana & 90 & 47.1 \\
\hline Total & 191 & \\
\hline
\end{tabular}

Apropiación de las principales tecnologías digitales (porcentaje de empleados que utilizan computadores)

Todos (100\%)

Más de la mitad (75\%)

$65 \quad 34.0$

La mitad (50\%)

20

10.5

Menos de la mitad (25\%)

41

21.5 
(Continuación)

\section{Variable}

N. R.

Total

Apropiacion
trabajo)

\begin{tabular}{|c|c|c|}
\hline Todos (100\%) & 64 & 33.5 \\
\hline Más de la mitad (75\%) & 52 & 27.2 \\
\hline La mitad (50\%) & 28 & 14.7 \\
\hline Menos de la mitad (25\%) & 43 & 22.5 \\
\hline N. R. & 4 & 2.1 \\
\hline Total & 191 & \\
\hline \multicolumn{3}{|l|}{ La empresa tiene sitio web } \\
\hline No & 63 & 33.0 \\
\hline Sí & 116 & 60.7 \\
\hline N. R. & 12 & 6.3 \\
\hline Total & 191 & \\
\hline \multicolumn{3}{|l|}{ Nombres de redes sociales virtuales que utiliza la empresa* } \\
\hline Facebook & 111 & 58 \\
\hline Twitter & 61 & 32 \\
\hline Google + & 58 & 30 \\
\hline Blogs & 16 & 8 \\
\hline YouTube & 39 & 20 \\
\hline ¿Otra? ¿Cuál? & 13 & 7 \\
\hline \multicolumn{3}{|l|}{ Tipo de medios de comunicación que utiliza la empresa* } \\
\hline Teléfono fijo (comunicaciones básicas) & 174 & 91 \\
\hline Teléfono móvil (comunicaciones básicas) & 166 & 87 \\
\hline Fax (comunicaciones básicas) & 131 & 69 \\
\hline Mensajería interna-intranet (comunicaciones avanzadas) & 103 & 54 \\
\hline Correo electrónico (comunicaciones avanzadas) & 170 & 89 \\
\hline (comunicaciones avanzadas) & 86 & 45 \\
\hline
\end{tabular}

*Preguntas de selección múltiple, múltiple respuesta. Cada recuento son los casos positivos en que se cumple la condición (sí/no) para el contacto total de 191 encuestas.

Fuente: Elaboración propia. 
Se observa en la tabla 5 que $57.6 \%$ de las empresas encuestadas pertenece al sector servicios, mientras que $23.6 \%$ realiza actividades de comercio, y $17.8 \%$ de transformación de materias primas. Respecto de la antigüedad de la empresa, 29.32 \% respondió que la empresa está en funcionamiento entre 10 y 25 años. Por su parte, $21.47 \%$ respondió que la empresa es relativamente nueva en el mercado al llevar menos de 5 años de antigüedad. Respecto del tamaño de la empresa, de las $191 \mathrm{em}$ presas encuestadas, 90 respondieron ser medianas, 65 pequeñas y $36 \mathrm{mi}$ croempresas. Respecto del porcentaje de empleados que utiliza computadores, $34 \%$ de las empresas encuestadas afirma que más de la mitad de sus empleados utilizan computadores para realizar las actividades diarias, muy seguido con $33 \%$ de empresas que manifiesta que $100 \%$ de sus empleados utiliza esta herramienta. Igualmente, respecto del porcentaje de empleados que utiliza internet en el trabajo, el más alto porcentaje es $33 \%$, y es el que explica que $100 \%$ de los empleados utiliza internet en el trabajo. En este sentido, no se exploraron las actividades para las cuales se utiliza internet; sin embargo, es probable que los empleados lo utilicen para otras cosas que afectan el desempeño laboral, como redes sociales, juegos en línea, entre otros.

Respecto del interrogante de si la empresa cuenta con sitio web, se encontró que 116 empresas (61 \%) responden que cuentan con página web; al explorar algunas de las páginas se encuentra que son páginas sencillas con contenidos básicos, como historia, productos, noticias e información de contacto.

En cuanto a la información de los tipos de medios sociales virtuales que utiliza la empresa, el encuestado podía seleccionar más de una respuesta; por lo anterior, los resultados se organizaron registrando los casos afirmativos en los que la empresa cuenta con cada condición investigada en las redes sociales. Así las cosas, $58 \%$ de los encuestados afirmó que la empresa utiliza Facebook; es común encontrar páginas de empresas en esta red social donde se realizan promociones, lanzamientos, descuentos, concursos, entre otros, quizás porque es la red social más utilizada en el mundo, con cerca de novecientos millones de usuarios registrados y por la facilidad de manejo (Gómez, 2012), ya que tiene una interfaz agradable e intuitiva que está al alcance de todos. En segundo lugar, las empresas reportan 
utilizar Twitter con $32 \%$, seguido de Google +, YouTube y blogs.

Al indagar los tipos de medios de comunicación que utiliza la empresa, se encontró que $91.09 \%$ utiliza telefonía fija, 86.91 \% telefonía móvil, porque las empresas de telefonía actualmente manejan paquetes especiales de descuento para empresas, y poco a poco va ganando importancia la utilización del sistema de voz IP, como Skype, ya que si la empresa cuenta con conexión a internet ilimitado puede utilizar este protocolo entre sedes, empleados o llamar a los clientes y proveedores de manera totalmente gratuita, siendo una propuesta alternativa para reducir costos.
Relaciones entre variables de caracterización de la empresa, uso de medios sociales virtuales y tipos de tecnologías de comunicación que utiliza la empresa

Para profundizar en el análisis de la caracterización y uso de medios sociales y de tecnologías de comunicación por parte de las pequeñas y medianas empresas en Bogotá, se realizó un análisis entre las variables objeto de investigación mediante el uso del estadístico chi-cuadrado. En este sentido, se lograron comprobar algunas relaciones, las cuales se han organizado en cuatro tablas según los intereses e hipótesis del estudio.

Tabla 6. Resultados estadísticos de asociación entre las variables de caracterización de la empresa y el uso de la empresa en medios sociales

\begin{tabular}{|c|c|c|}
\hline Cruces & $\begin{array}{c}\text { N. S. } \\
\text { (bilateral) }\end{array}$ & $\begin{array}{c}\text { N. conf. } \\
(\%)\end{array}$ \\
\hline Actividad de la empresa/redes sociales virtuales (Twitter) & $0.033^{*}$ & 95 \\
\hline Tamaño de la empresa/redes sociales virtuales (Facebook) & 0.098 & 90 \\
\hline Antigüedad de la empresa/la empresa tiene sitio web & $0.015^{*}$ & 95 \\
\hline Tamaño de la empresa/la empresa tiene sitio web & $0.000^{* *}$ & 99 \\
\hline Porcentaje de empleados que utilizan computadores/la empresa tiene sitio web & $0.012^{*}$ & 95 \\
\hline Porcentaje de empleados que utilizan internet en el trabajo/la empresa tiene sitio web & $0.023^{*}$ & 95 \\
\hline \multicolumn{3}{|l|}{ Otras relaciones de interés } \\
\hline Tamaño de la empresa/porcentaje de empleados que utilizan computadores & $0.007^{\star *}$ & 99 \\
\hline Tamaño de la empresa/porcentaje de empleados que utilizan internet en el trabajo & $0.000^{* *}$ & 99 \\
\hline Antigüedad de la empresa/porcentaje de empleados que utilizan computadores & $0.005^{* *}$ & 99 \\
\hline Antigüedad de la empresa/porcentaje de empleados que utilizan internet en el trabajo & $0.028^{*}$ & 95 \\
\hline Actividad de la empresa/porcentaje de empleados que utilizan internet en el trabajo & $0.004^{* *}$ & 99 \\
\hline
\end{tabular}

$p<0.1-p<0.05^{*}-p<0.01$ **

Fuente: Elaboración propia. 
En la tabla 6 se observa que, dentro de todos los cruces posibles de asociación realizados entre las variables, se lograron demostrar seis pares de asociaciones, en las cuales el valor $p$ fue inferior a los márgenes máximos de error permitidos. Se observa que el uso de Facebook por parte de las empresas (por ejemplo fan page, anuncios, páginas sugeridas) se encuentra asociado al tamaño de la empresa (en este caso, un mayor tamaño puede estar relacionado con la necesidad de mayor presencia en la web). Igualmente, existe una relación entre el uso de Twitter por parte de las empresas con la actividad de la empresa (siendo la actividad de servicios principalmente asociada al uso de Twitter, y la actividad de comercio, con su no uso).

Respecto de la disposición de la empresa de un sitio web, existe relación con las variables de antigüedad de la empresa, tamaño de la empresa, nivel de empleados que utilizan computadores y nivel de empleados que utilizan internet en el trabajo. De tal forma que existe mayor presencia de contar con un sitio web con las empresas que llevan más tiempo en el mercado, que su tamaño sea mayor y que tenga mayor cantidad de empleados que utilizan computadores y tienen acceso a internet en sus trabajos.

Sobre otras relaciones de interés que surgieron en el análisis de resultados, se encuentra que tanto el tamaño y la antigüedad de la empresa tienen una asociación con la tecnificación virtual a empleados (posibilidad de uso de computadores e internet en el trabajo). Las relaciones significaron que existe una tecnificación virtual a empleados en los tamaños de microempresa, seguido por la condición de mediana empresa. Igualmente, entre menos antigua sea la empresa (menos de cinco años), mayor porcentaje de empleados utilizan computadores e internet.

Finalmente, también se demostró una relación entre la actividad de la empresa y la apropiación de las principales tecnologías digitales en los empleados, siendo la actividad de servicios la condición donde existe mayor porcentaje de empleados que utilizan internet en el trabajo. 
Tabla 7. Resultados estadísticos de asociación entre las características de la empresa y el tipo de tecnologías de comunicaciones utilizadas en su gestión corporativa

\begin{tabular}{lcc}
\hline \multicolumn{1}{c}{ Cruces } & $\begin{array}{c}\text { N. S. } \\
\text { (bilateral) }\end{array}$ & N. conf. \\
\hline Antigüedad de la empresa/medios de comunicación (fax) & $0.045^{*}$ & 95 \\
\hline Tamaño de la empresa/medios de comunicación (teléfono móvil) & 0.070 & 90 \\
\hline Tamaño de la empresa/medios de comunicación (voz IP) & 0.091 & 90 \\
\hline La empresa tiene sitio web/medios de comunicación (mensajería interna-intranet) & $0.000^{* *}$ & 99 \\
\hline La empresa tiene sitio web/medios de comunicación (voz IP) & $.004^{* *}$ & 99 \\
\hline
\end{tabular}

$p<0.1-p<0.05^{*}-p<0.01^{* *}$

Se observan en la tabla 7 cinco relaciones estadísticas entre los tipos de tecnologías de comunicación utilizados por las empresas (comunicaciones básicas: fax y teléfono móvil; y comunicaciones en línea: mensajería interna, voz IP) y sus propios atributos. En este sentido, existe una asociación entre el uso de fax y la antigüedad de la empresa (principalmente en empresas que llevan más de diez años utilizando fax, $\mathrm{y}$ con menos de cinco años utilizando fax). Sobre el uso de teléfono móvil para gestionar comunicaciones en la empresa (clientes, proveedores, compañeros de trabajo), se observa una relación con la variable de tamaño de la empresa. En este sentido, se puede deducir que a mayor tamaño de la empresa (mediana) no existe utilización de teléfono móvil (en este caso, dicho resultado es confirmado por la prueba de los residuos tipificados corregidos, o residuos ajustados, el cual permite interpretar con precisión el significado de la asociación detectada (Rodríguez, 2004). Por lo anterior, se considera que la empresa mediana no tiene necesidad de utilizar el teléfono móvil debido a preferir otras herramientas de tecnologías de comunicación para sus clientes; en contraste, precisamente tecnologías en línea como telefonía voz IP, que en dicho caso también fue demostrada de manera significativa mediante la prueba de residuos corregidos.

Asimismo quedó demostrada la relación entre la disposición de la empresa de un sitio web y el uso de la mensajería interna y la telefonía voz IP, con lo que, en la medida en que las empresas incorporan tecnologías de comunicación, también incorporan mecanismos de visibilidad en internet. 
Tabla 8. Resultados estadísticos de asociación entre el nivel de apropiación de las principales tecnologías digitales (computadores e internet) y tipo de tecnologías de comunicaciones empresariales

\begin{tabular}{lcc}
\hline \multicolumn{1}{c}{ Cruces } & N. S. (bilateral) & N. conf. \\
\hline $\begin{array}{l}\text { Porcentaje de empleados que utilizan computadores/medios de comunicación } \\
\text { (correo electrónico) }\end{array}$ & 0.089 & 90 \\
\hline $\begin{array}{l}\text { Porcentaje de empleados que utilizan internet en el trabajo/medios de } \\
\text { comunicación (mensajería interna) }\end{array}$ & $0.001^{* *}$ & 99 \\
\hline $\begin{array}{l}\text { Porcentaje de empleados que utilizan internet en el trabajo/medios de } \\
\text { comunicación (voz IP) }\end{array}$ & $0.000^{* *}$ & 99 \\
\hline
\end{tabular}

$p<0.1-p<0.05^{*}-p<0.01^{\text {* }}$

En la tabla 8 se observa que exis- telefonía voz IP. Al respecto, se destaca te una relación entre el porcentaje de con un nivel de confianza esta última empleados que utiliza internet con las asociación, lo que supone el servicio tecnologías de comunicación de co- de telefonía voz IP como medio de rreo electrónico, mensajería interna y comunicación en línea empresarial.

Tabla 9. Resultados estadísticos de asociación entre variables de uso en medios sociales virtuales que utiliza la empresa y apropiación de las principales tecnologías digitales (posibilidad de uso de computadores e internet)

\begin{tabular}{lcc}
\hline \multicolumn{1}{c}{ Cruces } & N. S. (bilateral) & N. conf. \\
\hline $\begin{array}{l}\text { Porcentaje de empleados que utilizan internet en el trabajo/redes sociales } \\
\text { virtuales (Facebook) }\end{array}$ & 0.054 & 90 \\
\hline $\begin{array}{l}\text { Porcentaje de empleados que utilizan internet en el trabajo/redes sociales } \\
\text { virtuales (Twitter) }\end{array}$ & 0.017 & 95 \\
\hline $\begin{array}{l}\text { Porcentaje de empleados que utilizan internet en el trabajo/redes sociales } \\
\text { virtuales (blogs) }\end{array}$ & 0.097 & 90 \\
\hline $\begin{array}{l}\text { Porcentaje de empleados que usan internet en el trabajo/redes sociales } \\
\text { virtuales (YouTube) }\end{array}$ & 0.095 & 90 \\
\hline$p<0.1-p<0.05^{*}-p<0.01^{* *}$ &
\end{tabular}

$\mathrm{Al}$ analizar la apropiación de las principales tecnologías digitales (posibilidad de uso de computadores e internet a empleados) con el uso de la empresa de medios sociales, se observa en la tabla 9 que, dentro de todos los cruces posibles de asociación realizados entre las variables, se lograron demostrar cuatro pares de asociaciones en las cuales el valor $p$ fue inferior a los márgenes máximos de error permitidos. En la tabla 9 se observa que el uso de Facebook por parte de las empresas (por ejemplo fan page, anuncios, y páginas sugeridas) se encuentra asociado con la variable nivel de empleados que utilizan internet en el trabajo (en este 
caso, puede indicar que a mayor disposición de internet para empleados, mayor uso de los empleados de Facebook e igualmente mayor necesidad de uso de actividades de visibilidad mediante dicha red social).

$\mathrm{Al}$ examinar las relaciones con el uso de Twitter por parte de las empresas, se demostró una relación con la variable de nivel de empleados que utilizan internet en el trabajo (es decir, a medida que más empleados tengan la necesidad de uso de internet en la empresa, se manifiesta la tecnificación de esta, lo que supone el uso empresarial de Twitter).

$\mathrm{Al}$ analizar el comportamiento de uso de blogs por parte de una empresa, la única relación demostrada es con el nivel de empleados que utilizan internet en el trabajo; por consiguiente, la necesidad de contar con acceso y apropiación de las principales tecnologías a empleados (computador e internet) hace evidente que la compañía desarrolle también presencia en la web. El mismo comportamiento se registra en el uso de YouTube con el nivel de empleados que utilizan internet en la empresa.

\section{Discusión}

El trabajo realizado permite comprender en mayor medida el estado de la empresa colombiana respecto del uso de las tecnologías de comunicación (medios convencionales, como fax, teléfono fijo y móvil; y medios avanzados (en línea), como correo electrónico, intranet y voz IP) según la propia estructura de tamaño, sector al que pertenece y antigüedad en el mercado. Dichos hallazgos aportan información empírica y son consecuentes con la literatura al confirmar que prácticamente todas las empresas han desarrollado herramientas y adaptado su comunicación a los diferentes medios y entornos a los que han ido emergiendo (García, 2011). En consonancia con dichos argumentos, también los hallazgos demostraron que las empresas han ido fortaleciendo su presencia en la web y la visibilidad en medios sociales para favorecer su planes comerciales, siendo internet y el desarrollo de páginas web un elemento indispensable de comunicación con los clientes (García, 2011).

Entender los cambios en la apropiación de la tecnología digital en las empresas supone también analizar la oportunidad que dan las empresas a sus empleados de utilizar no solo 
computadores para sus actividades laborales, sino la oportunidad de conexión a servicios de internet. Tal situación de acceso y uso pudo ser medida en las encuestas, las cuales demostraron mayor equipamiento tecnológico y comunicacional para las empresas con mayor tamaño y antigüedad en el mercado. Los hallazgos confirman el supuesto de que a mayor tamaño de la empresa mayor nivel de apropiación de la tecnología y comunicación por parte de la empresa, que en dicho caso, respalda el fuerte interés de las empresas medianas y pequeñas por la comunicación en línea, donde están evolucionando hacia una gestión cada vez más sofisticada, con múltiples herramientas y plataformas digitales que les permiten sorprender y cautivar al usuario (García, 2011).

Para corresponder con los hallazgos ante las hipótesis propuestas, a continuación se describe cada una a manera de conclusiones.

Respecto de la hipótesis 1, relación entre las características de la empresa y el uso de la empresa de medios sociales, la investigación aportó evidencia empírica de su demostración. Se pudo comprobar que el tamaño de la empresa se encuentra relacionado con Facebook; esto supone que entre más grande sea la empresa mayor necesidad de visibilidad tiene en la web, por lo que tener una cuenta en Facebook (siendo el principal medio social en Colombia) es pertinente para cautivar diferentes grupos de usuarios de interés interconectados. Igualmente, las alternativas para pautar en dicha plataforma son más variadas en comparación con otros medios sociales identificados. En segundo lugar, los hallazgos demostraron que el tipo de sector en el que participa la empresa tiene una asociación con el uso de Twitter, siendo la actividad de servicios principalmente asociada a la misma red social, y la actividad de comercio, con la condición de no uso de Twitter. Estos resultados permiten inferir que las empresas de servicio están aprovechando las tecnologías de comunicación para obtener seguidores $y$, de esta forma, probablemente aumentar su familiaridad y posicionamiento en el mercado, lo que supone mayor exploración en próximas investigaciones. En cuanto a la antigüedad de la empresa y el uso de medios sociales, los resultados obtenidos no aportan información concluyente respecto de algún tipo de asociación; sin embargo, la disposición de una página web de la empresa sí se encuentra asociada 
a la mayor antigüedad de la empresa, mayor tamaño y mayor nivel de apropiación de tecnologías digitales (cantidad de empleados que utilizan computadores y tienen acceso a internet en sus trabajos).

Respecto de la hipótesis $2 \mathrm{a}$, relación entre las características de la empresa y el tipo de tecnologías de comunicaciones utilizadas en su gestión corporativa, la investigación también logra comprobarla mediante los resultados. La condición de antigüedad de la empresa superior a diez años estuvo asociada al uso de fax, mientras que las empresas de menos de cinco años se asociaron con la no utilización del mismo medio convencional, sin existir evidencia de utilización de tecnologías de comunicación en línea con dicho elemento empresarial. En contraste, el tamaño de la empresa estuvo asociado con la no utilización de teléfono móvil en sus tecnologías de comunicación corporativa. Esto permite suponer que las empresas entre más grandes sean mayor acceso a tecnologías de comunicación en línea poseen, ante lo cual excluyen medios convencionales de contacto, como el teléfono móvil; dicho argumento es igualmente sustentado por la relación de mayor tamaño de la empresa (mediana empresa) con la tecnología de comunicación de voz IP, con lo cual se percibe la sustitución. Finalmente, la presencia en internet mediante un sitio web estuvo asociada a las tecnologías de comunicación de mensajería interna y telefonía voz IP, lo cual refuerza la idea de que a mayor adopción de tecnología digital mayor es la necesidad de visibilidad de la empresa en internet.

Siguiendo el análisis anterior, la hipótesis 2 b, relación entre la apropiación de las principales tecnologías digitales y el tipo de tecnologías de comunicaciones utilizadas por las empresas en su gestión corporativa, también pudo ser validada. Los resultados demuestran una asociación entre las tecnologías de comunicación avanzada (en línea) de mensajería interna, correo electrónico y telefonía voz IP, con el nivel de uso de internet por parte de los empleados en una empresa. Dicho argumento es completamente lógico de esperar, pues supone que, en la medida de mayor acceso a computadores con internet, el uso de la comunicación en línea es más fuerte.

Respecto de la hipótesis 3, relación entre la apropiación de las principales tecnologías digitales y el uso de la empresa de medios sociales de comunicación, la investigación brinda resultados confirmatorios. Se observó 
que el uso de Facebook por parte de las empresas se encuentra asociado con la variable nivel de empleados que utiliza internet en el trabajo (en este caso, puede indicar que a mayor disposición de internet para empleados mayor necesidad y uso de la empresa de actividades de visibilidad en Facebook). Igualmente, la condición de uso de Twitter por parte de las empresas estuvo asociado con el nivel de empleados que utilizan internet en el trabajo. En complemento, la condición de uso de blogs y uso de YouTube por parte de una empresa también estuvo asociada con el nivel de empleados que utilizan internet en el trabajo.

Finalmente, la comprobación de las hipótesis brinda resultados alentadores sobre la adaptación de las empresas a las necesidades tecnológicas modernas en el campo de la comunicación y el marketing. En este sentido, la presencia de las empresas en internet supone la preparación y estructura necesaria para corresponder a las necesidades del mercado (mayor tamaño de la empresa, tipo de sector en el que participa y antigüedad). Dichas características pueden favorecer el tiempo de reacción y adaptación a los retos de la comunicación en línea y la propia complejidad y conductas de los usuarios interconectados.
Los nuevos retos de la comunicación en línea y de los medios sociales para la empresa suponen la creación continua de formas alternativas de contacto con los usuarios. En este caso, las actividades de visibilidad creativas, principalmente en Facebook (fan page, anuncios, páginas sugeridas) u otras actividades posibles, como el diseño de posts, presentaciones o, incluso, charlas o seminarios en línea, para favorecer la gestión corporativa y publicitaria

Como observaciones y limitaciones de este estudio, se espera enriquecerlo al incluir en investigaciones futuras ítems relacionados con las compras electrónicas a partir de las tecnologías en comunicación y medios sociales y el uso de dispositivos móviles como agentes en las nuevas formas de comunicación comercial en empresas. Dichas observaciones se encuentran justificadas en el crecimiento progresivo de las compras por internet, sistemas de pago en línea y aumento en la demanda de teléfonos inteligentes.

En conclusión, el estudio de las características de tecnologías de comunicación en línea y el papel de los medios sociales para favorecer programas de marketing deben ser entendidos como una oportunidad para 
las empresas. Las micro, pequeñas y medianas empresas pueden conseguir resultados similares a bajos costos con un mayor entendimiento de los recursos y las herramientas tecnológicos libres o licenciados, disponibles y adaptados al interés de cada empresa.

\section{Referencias bibliográficas}

Canals Margalef, J. (2001). La estrategia de la empresa en la era de internet. Información comercial española. ICE: Revista de Economía, 793, 57-76.

Colombia, DANE (2005). Censo general 2005. Recuperado de http://www. dane.gov.co/index.php?option=com content\&id=307\&Itemid=124

Cyr, D.; Kindra, G. y Dash, S. (2008). Web site design, trust, satisfaction and e-loyalty: the Indian experience. Online Information Review, 32(6), 773-790.

García Medina, I. (2011). Marketing digital multimedia: nuevos formatos $\mathrm{y}$ tendencias. Revista GEMINIS, año 2, 2, 37-45.

Ghose, S. y Dou, W. (1998). Internet functions and their impacts on the appeal of internet presence sites. Journal of Advertising Research, 38(2), 29-43.
Gómez Gallardo, M. B. (2012). Análisis del contenido de los grupos relacionados con la diabetes en la red social Facebook: posibilidades de intervención en el ámbito de la salud. Barcelona: Universitat Oberta de Catalunya. Colombia, DANE (2008). Informe de indicadores básicos de tecnologías de información y comunicación. Recuperado de http://www.dane.gov.co/ files/investigaciones/boletines/tic/ pres_tic_agos08.pdf

Klein, L. R. (2003). Creating virtual product experiences: the role of telepresence. Journal of Interactive Marketing, 17(1), 41-55.

Lichtenthal J. D. y Shay, E. (2003). Internet integration in business marketing tactics. Industrial Marketing Management, 32, 3-13.

Llano Naranjo, N. (2009). Política para la promoción en el acceso y uso de TIC en micro, pequeñas y medianas empresas colombianas. Bogotá: Ministerio de Comunicaciones. Recuperado de https://spi.dnp.gov.co/ App_Themes/SeguimientoProyectos/ ResumenEjecutivo/090518\%20-\%20 Politica\%20Uso\%20de\%20TIC\%20 
en\%20mipymes\%20-\%20MinComunicaciones.pdf

Mora, M. y Hurtado, C. (2011). ¿Cuáles son las percepciones, usos y hábitos de los colombianos sobre las tecnologías de la información y las comunicaciones? Recuperado de http:// www.mintic.gov.co/portal/604/w3article-2218.html

Rodríguez, J. R. (2004, octubre). Ayuda SPSS. Chi-cuadrado: notas metodológicas. Recuperado de http://www. rubenjoserodriguez.com.ar/wp-content/uploads/2011/06/Ayuda_SPSSChi_Cuadrado_Notas_Metodologicas.pdf

Palacio Beerli, A.; Santana, M. y Delia, J. (2010). La eficacia de la publicidad on-line en el contexto de los blogs. Cuadernos de Gestión, 10(1), 17-42.

Piñero, M. S. y Maya, S. R. de (2002). El consumidor ante las nuevas formas de comunicación comercial. Cuadernos Aragoneses de Economía,12(1), 97-110.

Colombia Navega. Reporte 2011-2012. Recuperado de http://blog.e-mipyme. com/2012/09/comportamiento-usuarios-en-linea-colombia-2011-2012.html
Rodríguez, A. G. (2012). La realidad de la pyme colombiana: desafío para el desarrollo. Recuperado de http:// www.fundes.org/uploaded/content/ publicacione/1241969270.pdf

Ryan, D. y Jones, C. (2009). Understanding digital marketing: marketing strategies for engaging the digital generation. Londres: Kogan Page.

Sandulli, F. D. etal. (2006). Uso de internet y paradoja de la productividad: el caso de las empresas españolas. Cuadernos de Economía y Dirección de la Empresa, 26, 149-173.

San José Cabezudo, R.; Gutiérrez Arranz, A. M. y Gutiérrez Cillán, J. (2004). Determinantes de la eficacia publicitaria del sitio web. Una aplicación del ELM. Revista Española de Investigación y Marketing, 8(2), 10-20.

Santomier, J. (2008). New media, branding and global sports sponsorship. International Journal of Sports Marketing and Sponsorship, 10(1), 15-28.

Sicilia, M.; Ruiz, S. y Munuera, J. L. (2005). Effects of intreractivity in a web site. Journal of Advertising, 34(3), 31-45. 
Simó, L. A.; Alcañiz, J. E. B. y Mafé, Vargo, S. L. y Lusch, R. F. (2004). C. R. (2004). Conceptualización del Evolving to a new dominant logic comportamiento del consumidor en for marketing. Journal of Marketing, los entornos virtuales: un análisis cua- $\quad 68(1), 1-17$. litativo. Estudios sobre Consumo, 70, 9-20.

Zott, C.; Amit, R. y Massa, L. (2011).

The business model: recent develop-

Valero, D. (2011). Internet pasaría a ments and future research. Journal of ser un derecho de todo ciudadano Management, 37(4), 1019-1042. en Colombia. Recuperado de http:// www.eltiempo.com/politica/internetpasaria-a-ser-un-derecho-de-todociudadano_10239829-4 